\title{
Bayesian Updating in Property Protection System
}

\author{
Katarína Kampová, Tomáš Loveček \\ Faculty of security engineering, University of Zilina \\ Univerzitna 8215/1, 01026 Zilina \\ Slovakia
}

Received: July 30, 2021. Revised: November 12, 2021. Accepted: December 5, 2021.

Published: December 30, 2021.

\begin{abstract}
The article focuses on the implementation of the Bayesian updating method in creating a model of the protection system. This implementation makes it possible to incorporate the new information obtained during the lifetime of the protection system. The inclusion of new information ultimately makes it possible to refine the results of the model and thus increase its effectiveness to support decision-making. The approach of using the Bayesian updating method is given in a specific example. The possibility of expert estimation of input parameter values and application of Bayesian updating for the purposes of quantitative revision of security level assessment is also presented.
\end{abstract}

Keywords-Security, Bayesian updating, property protection system.

\section{INTRODUCTION}

The model of property protection system is a complex probabilistic model based on the interrelationships of various parameters. These relationships are characterized by the fact that we do not know their actual values, respectively the observable actual values are characterized by significant randomness [1], [2], [3]. The parameters of the protection system model are therefore uncertain. Uncertainty is usually described in the form of subjective probabilistic estimates of the actual values of random events that model the parameters. The subjective way of interpreting probability is the basis of the Bayesian inference, often called "Bayesian probability"[4].

Based on this approach, it is possible to evaluate and assess any uncertainty of the parameters of the model of property protection system. However, the Bayesian interpretation not only brings to the concept of probability the dimension of subjectivism, but an important aspect of this approach is that it assumes that what it is reasonable for the expert to believe depends not only on estimation but also on observable experience [5], [6].

However, the Bayesian interpretation does not only bring a dimension of subjectivism to the concept of probability. An important aspect of this approach is that it assumes that what is reasonable for an expert to believe depends not only on estimation but also on observable experience. The expert's estimate is thus conditioned by the extent of the information available to him/her and therefore the subjective probability changes over time with the change in the available information based on empirical observations [7], [8]. The basic concept of the Bayesian updating is a rationally based revision of beliefs based on empirically obtained new information and observations of the estimated situation. This concept can be used for a systematic process of improving property protection system models and keeping them valid in the light of new relevant information [9], [10].

\section{BAYESIAN UPDATING AND PROPERTY PROTECTION SYSTEMS}

The theoretical basis for the use of Bayesian updating methods in probabilistic models is based on Bayes' theorem. The Bayes' theorem describes the relationship between the interdependence of a random event $A$ and the occurrence of one of the disjoint random events $B_{i}$ (these events are also referred to as hypotheses).

In a random experiment, exactly one of the hypotheses $B_{1}, B_{2}, \ldots, B_{n}$ occurs, whose probabilities $P\left(B_{i}\right)$ are known before the experiment is performed. This is the so-called a priori probabilities. Information on whether phenomenon $A$ has occurred on the basis of the experiment changes the probabilities of the individual alternatives from $P\left(B_{k}\right)$ to $P\left(B_{k} \mid A\right)$. The probabilities $P\left(B_{k} \mid A\right)$ are called posterior [11]. The way in which these probabilities are calculated (1) is defined by Bayes' theorem. 
$P\left(B_{k} \mid A\right)=\frac{P\left(B_{k}\right) P\left(A \mid B_{k}\right)}{\sum_{i=1}^{n} P\left(B_{i}\right) P\left(A \mid B_{i}\right)}=\frac{P\left(B_{k}\right) P\left(A \mid B_{k}\right)}{P(A)}$.

The Bayesian updating method can be generalized and extended by a straight-line application to a random variable and its distribution function. The Bayesian updating can thus be used through a combination of the priori distribution function and the likelihood function to create the posteriori distribution function [12]. The relation defines the use of Bayes' theorem to update a distribution function:

$$
f(\theta \mid H)=f(\theta) L(H \mid \theta) c .
$$

The function $f(\theta)$ represents the priori function of the probability distribution of the random variable $\theta$. This function is derived from expert judgement and represents a subjective degree of belief in the probability distribution of the examined random variable $\theta$. The posterior distribution function $f(\theta \mid H)$ is an update of the probability distribution function of the random variable $\theta$ based on the set of observations $H$ of the actual value of the variable $\theta$.

The plausibility function $L(H \mid \theta)$ defines the probability with which the observation $H$ occurs. It is assumed that the real system that generates observable values is controlled by the parameter $\theta$ and the function describes the regularity of the randomness of the system with which this system creates variability of individual observations. This function therefore represents a modelled parameter of the property protection system, the values of which are implied by the random variable $\theta$.

The normalization constant $c$ corresponds to the inverse value of the probability of obtaining the set of observations $\mathrm{H}$. The normalization constant is defined by the integral of the product of the priori distribution function and the plausibility function [12]. Application of the Bayesian updating method is suitable for use in cases where we analyze and describe a stochastic system, or its quantity, which acquires observable random values. The uncertainty of such a system parameter can be described by assumptions about the probability distribution class, which govern the occurrence of observable values of the parameter. The function of this probability distribution is a function of plausibility. Although we have an assumption about this function, for example that it is a Poisson probability distribution, we do not know the exact value of some characteristic of this distribution, for example the parameter $\lambda$ in the case of the Poisson distribution [10]. This characteristic is therefore an uncertain parameter.

We can eliminate the uncertainty of this parameter by expressing a subjective assumption about the probability of which the parameter $\lambda$ will acquire what values. This assumption is written in the form of the priori probability distribution function. Subsequently, when real value observation is available, we can use the Bayesian updating to reevaluate our assumption about the probability distribution parameter in the background of this system and change the belief about the probability distribution of this parameter in the form of the posteriori probability distribution function [4].
For example, we have the parameter $x$ of the property protection system model, which is governed by a normal distribution with an indefinite mean value $\mu$ and a variance $\sigma^{2}=2$. We describe the uncertainty of the mean value $\mu$ with a random variable $\theta$. We assume acquires values according to the normal distribution with a mean value of 6 and a unit variance. The parameter $\mathrm{x}$ is described by the plausibility function $f(x \mid \theta) \sim N(\mu, 2)$ and the hyperparameter $\mu$ by the priori probability distribution function $f(\theta) \sim N(6,1)$. Subsequently, we obtain a real observation whose value is equal to 13. Based on this observation, we can adjust our assumption about the probability distribution function of the random variable $\theta$.

In other words, based on the empirical observation of the manifestation of the real system, we can revise the model of the property protection system in an exact way. This revision (3) consists in changing the priori function of the probability distribution $f(\theta)$ to the posteriori function $f(\theta \mid x)$, where $x$ represents the real observed value. We determine the posteriori function $f(\theta \mid x)$ as the product of the priori function and the plausibility function:

$$
f(\theta \mid x) \propto f(\theta) f(x \mid \theta) \propto e^{\frac{-3 \theta+2(12+x) \theta}{4}}
$$

When we substitute the observed value $x=13$ into the expression, we can determine that the posteriori distribution is a normal distribution with a mean value of $\mu \approx 8,333$ and a variance of $\sigma^{2}=0,667$. By introducing empirically obtained information, we were able to mathematically substantiate the shift in our belief in the probability distribution of the parameter $\theta$. This shift is shown in the following figure (Fig. $1)$.

In the given example, the calculation of the posteriori distribution function was simplified by omitting the

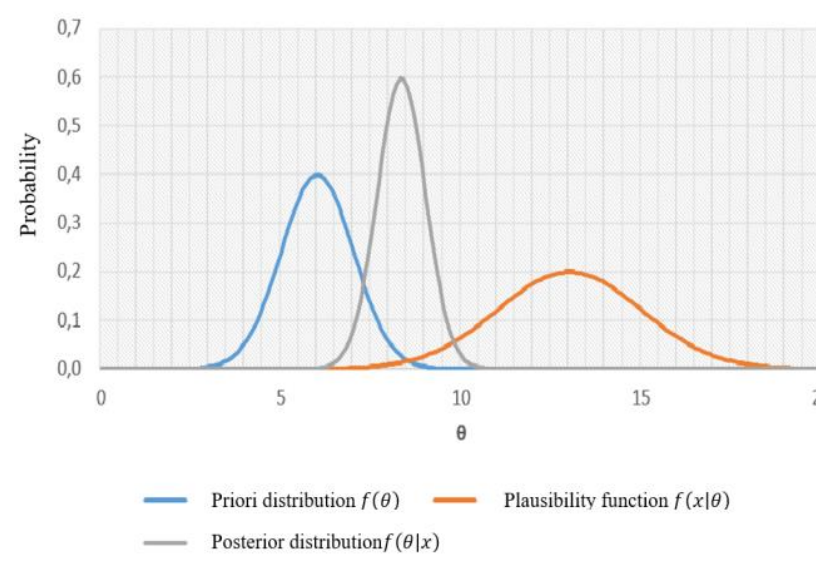

Fig. 1. Bayesian updating of the parameter estimate $\theta$.

normalization constant (4), calculating the probability proportionality, and then skipping to the resulting probability distribution. In general, however, it is the calculation of the normalization constant that most complicates the application 
of the Bayesian updating in the context of probability distribution functions. The normalization constant, which is based on the principle of the total probability theorem, has the following form:

$$
c=\frac{1}{\int L\left(H \mid \theta^{\prime}\right) f\left(\theta^{\prime}\right) d \theta^{\prime}} .
$$

The type of the priori distribution function $f\left(\theta^{\prime}\right)$ affects the algebraic form of the product $L\left(H \mid \theta^{\prime}\right) f\left(\theta^{\prime}\right)$ and how complicated this product is to calculate analytically [13]. Some specific types of the priori probability distribution with respect to the type of plausibility function distribution produce the same algebraic form of the posteriori distribution in this product. If priori and posteriori probability distributions have the same type, then we speak of a conjugate distribution function and a conjugated priori function, respectively.

The conjugated priori function creates the posteriori function in the form of a mathematical expression that is analytically solvable by a finite number of steps. It allows solving the integral of the normalization constant analytically and it is not necessary to use numerical procedures [14].

In finding the appropriate type of conjugated priori probability distribution, we must realize that in this we do not have complete freedom to choose any priori distribution that would work mathematically, but we must define the priori distribution that adequately describes subjective knowledge of an indefinite parameter before obtaining real observation.

Thus, the type of the priori conjugate probability distribution must be flexible enough to produce, on the one hand, a practical, analytically simple solution to the integration problem and, on the other hand, to model our priori degree of belief in parameters of interest [13]. This limitation thus reduces the possibilities of implementing the Bayesian updating into software tools and their use in asset protection systems [14].

Numerical methods, in contrast to the analytical mathematical procedures in solving the Bayesian updating, provide a different view of this issue and create space for alternative procedures in the case of some limitations. The basic starting points of the Bayesian updating method remain valid and the principles of interpretation of uncertainty of model parameters do not change either.

With the numerical solution, we can gain more freedom in expressing the uncertainty of these parameters [15]. Since the choice of appropriate types of probability distributions does not complicate the solution of integration of complete probability in the denominator of the Bayesian formula.

This is solved numerically and therefore the choice of the type of the priori distribution function is not limited by the exact solvability of the mathematical expression and thus more accurately reflects the current degree of belief in the probability distribution of the modelled parameter.

The resulting the posteriori function consequently does not necessarily have a standard type of distribution but is expressed by enumerating the values of the parameter with appropriate probabilities.
Numerical methods necessarily involve the involvement of software tools, through which the calculation itself is performed [14]. These are a limiting aspect of the application of numerical procedures, as especially more complex models and related analytical requirements require complex specialized or proprietary software solutions adaptable to user functional requirements. However, for a simple application, or an application for a partial or simplified part of the model, commonly available spreadsheets are also sufficient.

On the other hand, software solutions, in addition to the calculation itself, often allow to easily visualize the calculated uncertainty of parameters and thus bring another dimension to understanding the modelled system and subsequently support decision-making based on its outputs [10]. These solutions in the numerical processing of the Bayesian updating method represent uncertainties of model parameters in the form of enumeration probability distributions [11]. This form is directly applicable in simulation tools that support the processing of model outputs and are often directly integrated into software solutions.

\section{EXAMPLE OF USING THE BAYESIAN UPDATING WITHIN PROPERTY PROTECTION SYSTEMS}

The protection system model is a complex probabilistic model based on the interrelationships of various parameters, which are characterized by the fact that we do not know their actual values [16], respectively the observable actual values are characterized by randomness. The parameters of the protection system model are therefore uncertain, and this uncertainty is described in the form of probabilistic estimates of the actual values of the random phenomena that the parameters model.

The model compiled in this way provides several possibilities for the application of the Bayesian updating. Based on a new empirical observation of the actual values of the model parameters, it is possible to incorporate new available information into the model and update the priori probabilistic assumptions of the model.

Bayesian updating method allows the model to be gradually improved to interpret the modelled reality more accurately [4]. The method of using the Bayesian updating method is shown on the example of one variable model. This variable is, for example, the time of overcoming the door panel in the building on the offender's critical path (edge between points A and B in Fig.2).

Assume that the overcoming time was defined by an average time of 105 seconds (in the case of finding the shortest path in the graph), or by the normal distribution of $\mathrm{N}$ $(105,20)$ in the case of determining the output parameters of the model. If the quantification of the parameters of the distribution enters the model based on expert judgment, then the determination of the exact value narrows the possibilities of expressing the degree of belief of experts and thus distorts the interpretation of their expert view. 


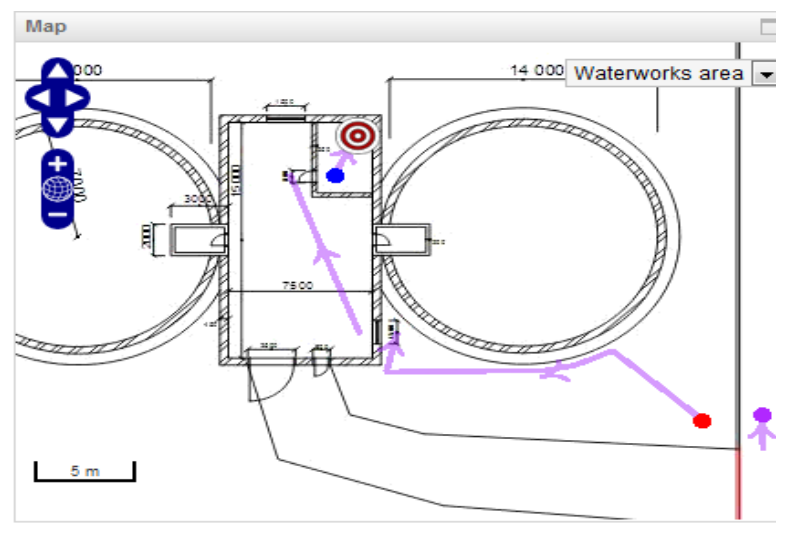

Fig. 2. Illustration of overcoming a door

If we approach the description of these parameters of the distribution as a random variable, then on the one hand it allows to reliably interpret the degree of belief of experts in its full breadth and on the other hand allows updating this belief by incorporating empirical observation as soon as it becomes available. In this case, the parameter is modelled by the variable $X \sim N\left(\mu_{x}, \sigma_{x}^{2}\right)$. For simplicity, we will consider the constant standard deviation $\sigma_{\mathrm{x}}=20$.

The parameter of the distribution $\mu_{x}$, which we also refer to as the hyperparameter, will not be constant compared to the previous approach, but will allow expressing the full degree of conviction of experts about its probable value. The hyperparameter $\mu_{x}$ is thus a random variable, the probability distribution of which can be expressed, for example, by the following enumeration of hypotheses (Table 1.).

TABLE I. PRESENTATION OF THE HYPERPARAMETER

\begin{tabular}{|c|c|c|}
\hline $\begin{array}{c}\text { Hypothesis } \\
\mu_{\mathbf{x}}\end{array}$ & $\begin{array}{c}\text { Hyperparameter value } \\
\mu_{\mathbf{x}}\end{array}$ & $\begin{array}{c}\text { Probability of the hypothesis } \\
\mathbf{P}\left(\mathbf{B}_{\mathbf{k}}\right)\end{array}$ \\
\hline $\mathbf{B}_{\mathbf{1}}$ & 85 & 0,2 \\
\hline $\mathbf{B}_{\mathbf{2}}$ & 100 & 0,4 \\
\hline $\mathbf{B}_{\mathbf{3}}$ & 110 & 0,3 \\
\hline $\mathbf{B}_{\mathbf{4}}$ & 125 & 0,1 \\
\hline
\end{tabular}

The above distribution of the probability with which the hyperparameter $\mu_{x}$ acquires values may be the result of expert assessment of several experts, expressing a combination of their expert views, or it may be a combination of assessment of alternative conditions of overcoming a barrier, or an expression of one expert's belief in the expected value of hyperparameter $\mu_{x}$.

In the same way, it is possible to determine the hyperparameters of another probability distribution attempt that model phenomena in the property protection system. The parameter of the property protection system model $X \sim N\left(\mu_{x}, \sigma_{x}^{2}\right)$ defined in this way with the hyperparameter $\mu_{x}$ determined by the probability distribution according to Table 1 can be processed by means of various simulation methods. If we obtain a real observation of the value of the parameter $X$, we need to perform a revision of the degree of belief in the probability distribution of the values of the hyperparameter $\mu_{x}$, we need to perform a revision of the degree of belief in the probability distribution of the values of the hyperparameter $\mu_{x}$.

The initial (priori) probability $P\left(B_{k}\right)$ changes to the posteriori probability $P\left(B_{k} \mid A\right)$ based on the observation of the phenomenon $A$ (empirically determined value of the parameter of the model $X$ ). The Bayesian updating is used for this change. Assume that a time of 118 seconds was found in the barrier test. To be able to incorporate this information into the model through the Bayesian updating, it is necessary to determine the likelihood $P\left(A \mid B_{k}\right)$ and the complete probability $P(A)$, respectively normalization constant $\frac{1}{P(A)}$.

Plausibility (5) describes the stochastic manifestations of the $X$ parameter of the model by defining the probability with which the $X$ parameter (barrier time) will reach the observed value of 118 seconds if the $X$ parameter has a normal distribution with the hyperparameter value $\mu_{x}$ according to the $B_{k}$ hypothesis [9]. We will use the plausibility function for practical calculation:

$$
f\left(x \mid \mu_{k}\right)=\frac{1}{\sigma \sqrt{2 \pi}} e^{-\frac{\left(x-\mu_{k}\right)^{2}}{2 \sigma^{2}}} .
$$

In the plausibility function $f\left(x \mid \mu_{k}\right)$, the standard deviation $\sigma=20$ is constant, the mean value depends on the hypothesis $B_{k}$, and $\mathrm{x}=118$ represents the observed value. The complete probability $P(A)=\sum_{k=1}^{4} P\left(B_{k}\right) P\left(A \mid B_{k}\right)$ is an expression of the possibility that the observed phenomenon A will occur, (overcoming time will be 118 seconds) in the context of the modeled parameter $X$, for example $P(A)=P(X=118)$. The value of $P(A)$ is determined by an iterative calculation through individual hypotheses $P\left(B_{k} \mid A\right)$ and (1).

Based on this calculation, we expressed the change in the belief about the possibility with which the hyperparameter $\mu_{k}$ will acquire its values as follows (Table 2).

TABLE II. CHANGE OF BELIEFS ABOUT HYPERPARAMETERS $\mu_{k}$

\begin{tabular}{|c|c|c|c|}
\hline $\begin{array}{c}\text { Hypothesis } \\
\mu_{\mathbf{x}}\end{array}$ & $\begin{array}{c}\text { Hyperparameter value } \\
\mu_{\mathbf{x}}\end{array}$ & $\begin{array}{c}\text { Priori probability } \\
\mathbf{P}\left(\mathbf{B}_{\mathbf{k}}\right)\end{array}$ & $\begin{array}{c}\text { Posteriori probability } \\
\mathbf{P}\left(\mathbf{B}_{\mathbf{k}} \mid \mathbf{A}\right)\end{array}$ \\
\hline $\mathbf{B}_{\mathbf{1}}$ & 85 & 0,2 & 0,074 \\
\hline $\mathbf{B}_{\mathbf{2}}$ & 100 & 0,4 & 0,387 \\
\hline $\mathbf{B}_{\mathbf{3}}$ & 110 & 0,3 & 0,402 \\
\hline $\mathbf{B}_{\mathbf{4}}$ & 125 & 0,1 & 0,137 \\
\hline
\end{tabular}

This change in the probability space distribution of hypotheses $B_{k}$ is reflected in the distribution of the statistical file that is generated in the model of property protection systems for parameter $\mathrm{X}$ (for example when using simulation methods). By updating the probability space distribution of hypotheses about the hyperparameter $\mu_{k}$, the probability distribution of the parameter $\mathrm{X}$ was changed.

Fig. 3 shows an updated histogram based on the posterior distribution of $P\left(B_{k} \mid A\right)$. From the comparison, the shift caused by the empirical observation of the value of the parameter $\mathrm{X}=118$ seconds can be seen, which was reflected in the overall tendency to generate higher values of the model 
parameter

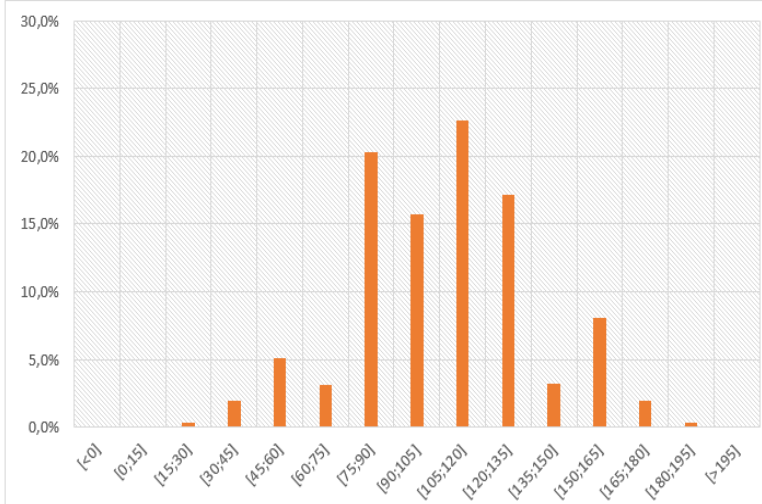

Fig. 3. Histogram of the posterior distribution of parameter $X$.

The values of the parameter $X$ generated based on the updated probability distribution determined by changing the priori probability space of the experts' hypotheses can then be used directly in the processing of the model by simulation methods. In this way, it is possible to achieve a rational revision of the model of the property protection system model in the context of new information obtained over time and to specify the output parameters of the model determining the evaluation of the effectiveness of the entire system.

\section{CONCLUSION}

In the article, we presented the possibilities of using the Bayesian updating method in property protection models. We described the basis of this method based on Bayes' theorem and we pointed out the possibilities of its use as a tool that integrates a subjective view of the priori probability, interpreted as a function of degree of belief and empirical information about the true value of the modeled parameter. In the article, we also presented the possibility of practical use of this method on a specific example.

\section{ACKNOWLEDGMENT}

The article was supported by The Ministry of Education, Science, Research and Sport of the Slovak Republic and Slovak research and development agency grant number APVV20- 0457.

\section{REFERENCES}

[1] L. Lukas, M. Hromada, "Simulation and Modelling in Critical Infrastructure Protection," International Journal of Mathematics and Computers in Simulations, pp. 386-394, 2011.

[2] M. Boros, F. Lenko, "Possibility of Transmission System Disruption by Intruder," in 13th International Scientific Conference on Sustainable, Modern and Safe Transport 2019, 40, pp. 1266-1272.
[3] M. Boros, A. Velas, V. Soltes, J. Dworzecki, "Influence of the Environment on the Reliability of Security Magnetic Contacts," Micromachines, Vol. 12, Iss. 4., 2021.

[4] T. Aven, Foundations of Risk Analysis, Chichester: John Wiley and Sons 2012.

[5] M. Boros, Z. Zvakova, M. Halaj, "Required competencies of security managers for decision-making," in 13th International Technology, Education and Development Conference (INTED), pp. 3918-3923, 2019.

[6] A. Siser, L. Maris, D. Rehak, W. Pellowski, "The use of expert judgement as the method to obtain delay time values of passive barriers in the context of the physical protection system," in 52nd Annual IEEE International Carnahan Conference on Security Technology 52, pp. 126-130, 2018.

[7] K. Kampová, "Expert judgement within the framework of risk assessmant of industrial processes," in Recent advaces in industrial and manafuacturing technologies. Athens: Vouliagmeni, pp. 216-220, 2013.

[8] A.M. Hanea, G.F. Nane, "Calibrating experts' probabilistic assessments for improved probabilistic predictions", Safety Science, 118, pp. 763-771, 2019.

[9] D. Vose, Risk Analysis: A Quantitative Guide. Chichester: John Wiley \& Sons, 2008.

[10] T. Avent, Misconceptions of Risk. Stavanger: University of Stavanger, 2010.

[11] S. Ferson, "Bayesian Methods in Risk Assessment.", Available: https://www.researchgate.net/publication/228805839_Bayesian Methods_in_Risk_Assessment.

[12] $\bar{S}$. C. Hora, "Eliciting Probabilities form Expert," in W. Edwards, R. F. Jr., \& D. v. Winterfeldt (Ed.), Advances in Decision Analysis. Cambridge University Press, pp. 129-153, 2007.

[13] D. Fink, "A Compendium of Conjugate Priors," Bozeman: Montana State Univeristy, 1997.

[14] D. W. Hubbard, The Failure of Risk Management. New Jersey: John Wiley \& Sons, 2009.

[15] A. T. Zagorecki, D. E. A. Johnson, J. Ristvej, "Data Mining and Machine Learning in the Context of Disaster and Crisi Management," International Journal of Emergency Management 9, pp. 351-365, 2013.

[16] M. Boros, M. Halaj, A. Velas, "Possibility of Transmitting Alarm Messages over the Radio Network to the Monitoring and Alarm Receiving Centre," in CBU International Conference Proceedings: innovations in science and education 6, pp. 10131016, 2018

\section{Author Contributions:}

Katarína Kampová focused on the application of the Bayesian updating to the property protection system. (III, IV).

Tomáš Loveček focused on theoretical basis of Bayesian updating and the issue of property protection system (I, II.).

\section{Creative Commons Attribution License 4.0 (Attribution 4.0 International, CC BY 4.0)}

This article is published under the terms of the Creative Commons Attribution License 4.0 https://creativecommons.org/licenses/by/4.0/deed.en US 